\title{
The Policy Machine for Security Policy Management
}

\author{
Vincent C. Hu, Deborah A. Frincke, and David F. Ferraiolo \\ National Institute of Standards and Technology, 100 Bureau Dr. Stop 8930 Gaithersburg \\ Maryland 20899-8930, USA \\ \{vhu, dferraiolo\}@nist.gov \\ Department of Computer Science of the University of Idaho, Moscow Idaho 83844, USA \\ frincke@cs.uidaho.edu
}

\begin{abstract}
Many different access control policies and models have been developed to suit a variety of goals; these include Role-Based Access Control, Onedirectional Information Flow, Chinese Wall, Clark-Wilson, $\boldsymbol{N}$-person Control, and DAC, in addition to more informal ad hoc policies. While each of these policies has a particular area of strength, the notational differences between these policies are substantial. As a result it is difficult to combine them, both in making formal statements about systems which are based on differing models and in using more than one access control policy model within a given system. Thus, there is a need for a unifying formalism which is general enough to encompass a range of these policies and models. In this paper, we propose an open security architecture called the Policy Machine $(P M)$ that would meet this need. We also provide examples showing how the $P M$ specifies and enforces access control polices.
\end{abstract}

\section{Introduction}

Access control is a critical component of most approaches to providing system security. Access control is used to achieve three primary objectives: (1), determining which subjects are entitled to have access to which objects (Authorization); (2) determining the access rights permitted (a combination of access modes such as read, write, execute, delete, and append); and (3) enforcing the access rights. An access control policy describes how to achieve these three goals; to be effective, this policy needs to be managed and enforced. There is a vast array of techniques that define and enforce access control policies within host operating systems and across heterogeneous bodies of data [NCSC98]. Although these techniques are successful in the specific situations for which they were developed, the current state of security technology has, to some extent, failed to address the needs of all systems [Spencer et al99, HGPS99] in a single notation. Access control policies can be as diverse as the applications that rely upon them, and are heavily dependent on the needs of a particular environment. Further, notations that easily express one collection of access control policies may be awkward (or incapable) in another venue. An example of this situation would be when a company's documents are under One-direction Information Flow [BL73, Biba77, Sand93] policy control at the development stage. When the development is finished,

V.N. Alexandrov et al. (Eds.): ICCS 2001, LNCS 2074, pp. 494-503, 2001.

(C) Springer-Verlag Berlin Heidelberg 2001 
the documents that are available for use by employees, could then be required to be controlled by a role-based or RBAC [FCK95, SCFY96] policy. Most existing commercial technologies used to provide security to systems are restricted to a single policy model, rather than permitting a variety of models to be used [Spencer et al99]. For instance, Linux applies a DAC [NCSC87] policy, and it is difficult to implement RBAC policy (among others) in such a system. Further, if an organization decides to change from one policy model to another, it is quite likely that the new policy model will have to be implemented above the operating systems level, perhaps even as part of the application code or through an intermediary. This is inconvenient, subject to error, slow, and makes it difficult to identify or model the overall "policy" that is enforced by the system.

To meet this challenge, we have developed the Policy Machine (PM). The underlying concept of the $P M$ relies on the separation of the access control mechanism from the access control policy [JSS97, JSSB97]. This enables enforcement of multiple access control policies within a single, unified system. Although complete policy coverage is an elusive goal, the $P M$ is capable of expressing a broad spectrum of wellknown access control policies. Those we have tested so far include: One-directional Information Flow, Chinese Wall [BNCW89], N-person Control [NCSC91] and DAC. These were selected partly because they are so well known, and partly because they differ greatly from one another. A further advantage of $P M$ is that it is highly extensible, since it can be augmented with any new policy that a specific application or user may require. This paper will demonstrate the functionalities of $P M$, and illustrate $P M$ 's universal, compositional and combinational properties.

\section{Policy Machine (PM)}

In this section we describe our design of $P M$, and explain how we achieve our goal of a unified description and enforcement of policies. Our design of $P M$ is related to the traditional reference monitor approach. A reference monitor is not necessarily a single piece of code that controls all accesses; rather, it is an abstraction or model of the collection of access controls [Ande72]. As we have applied the concept, $P M$ does not dictate requirements for particular types of subject or object attributes nor the relationships of these attributes within its abstract database. Because of this generality $P M$ can be used to model the implementation of a wide variety of access control policies, but $P M$ is not itself a policy.

The structure of $P M$ is based on the concept that all enforcement can be fundamentally characterized as either static (e.g., BBAC), dynamic (e.g., Work Flow), or historical (e.g., Chinese Wall) [GGF98, SZ97]. In the next five sections, we introduce the primary components of the PM: the Policy Engine ( $P E)$ with $P E$ databases and the security policy operations, which is the General Policy Operations (GPO). We then discuss the Universal and Composition properties of the $P M$ following each introduction. 


\subsection{Policy Engine (PE)}

The task of the $P E$ is to receive access requests and determine whether they should be permitted. Access requests are of the form $<$ User Identity, Requested Operations, Requested Objects $>$. To determine the acceptability of a request, the $P E$ executes three separate phases (Figure 1): the request management phase, the subject-object mediation phase, and the access history recording and database management phase to determine whether access is granted or denied. The request management phase is primarily used to assign the requesting user to a subject. The subject-object mediation phase is used to decide if the subject is permitted to access the requested object. The access history recording and database management phase determines if the granted access will affect the state of the $P M$ if a historical policy is implemented.

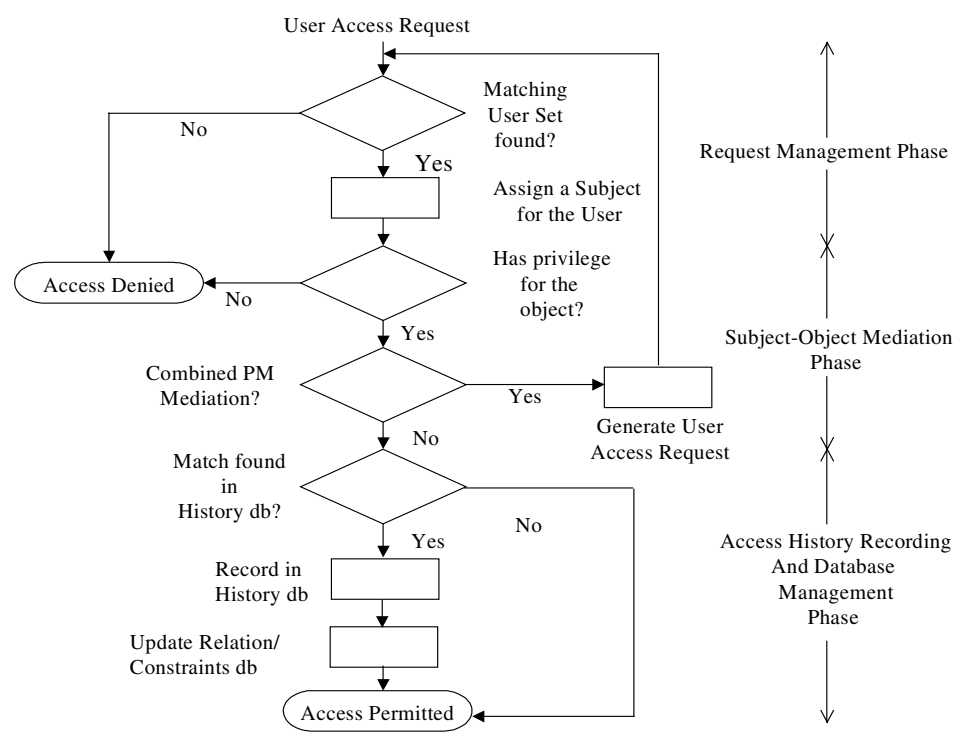

Fig. 1. $P M$ phases

\section{Request Management Phase (RMp)}

This phase is activated by the detection of a user request. The $P E$ responds by creating a subject along with operation, and object specified within the user's request. For example, consider the following request:

(Mary; write; a_file) i.e., user Mary wants to write to a_file. Currently, the Relations database ( $R d b)$ has entries as follow:

Managers $=($ Mary $)$, Programmers $=($ Managers, Bob, Sue $)$ i.e., the Managers user set contains user Mary, and user set Programmers contains user set Managers and users Bob and Sue.

Thus, user Mary is assigned as:

subject $=($ Mary, Managers, Programmers); write; a_file

and is able to continue to the next phase. 


\section{Subject-Object Mediation Phase (SOMp)}

The SOMp takes the input (subject, operations and object) created by the $R M p$, and the process authorizes access under two conditions. First, there is a match of the subject's request and there is an entry in the Privilege database $(P d b)$. In this case, the requested access is authorized. Second, there exists no further subject-object mediation check that is required under a different $P M$. For example, assume that, in the $S O M p$,

$P E$ generated the following message when in the $R M p$ :

subject $=($ Mary, Managers, Programmers); write; a_file

The $S O M p$ will then check $P d b$, and find entries:

(Programmers; all; a_file)

This means that the Programmers user set has all privileges to the file a_file. Therefore the request

subject $=($ Mary, Managers, Programmers); write; a_file

is authorized.

\section{Access History Recording and Database Management Phase (AHRDMp)}

This phase evaluates the relevance of the authorized event with respect to the historybased policies that are stored in the History Based Relations database (HBRdb). History-based policies are driven by an event and an action stored in the HBRdb. If the event received matches events stored in the HBRdb then PE in AHRDMp invokes the action associated with the event. The action either creates new constraints in the Constraints database $(C d b)$, and/or updates relations in the $P d b$. Consistency is checked whenever a relation or constraint is created. For example, $H B R d b$ contains an entry:

event $=$ (subject_a; all; $x_{-}$file), response $=($Generate Constraints $=$ (((subject_a; all; y_file) $\left.\oplus\left({ }^{*}::^{* *}\right)\right)$, ((subject_a; all;z_file) $\left.\left.\oplus\left({ }^{*}: *^{* *}\right)\right) ; C d b\right)$

This means that subject_a is prohibited from any kind of access (by excluding $(\oplus$ ) all (*)) to $y_{-}$file and $z$ file if $x_{-}$file has been accessed by subject_a. Entries for the constraints will be added into the $C d b$. This example can be used for policies that require SOD constrains, such as Chinese Wall [BNCW89] policies.

\subsubsection{PE Databases}

$P E$ databases provide relation, privilege, constraint, and history information for $P E$ processes. The databases are maintained either by GPO or by the AHRDMp. In this section, we will describe each of the four types of databases.

Relations database (Rdb) maintains the inheritance relations between sets. For example, $R d b$ is used in the $R M p$ to establish the active user set( $(s)$ of a subject. An example is in Figure 2. 


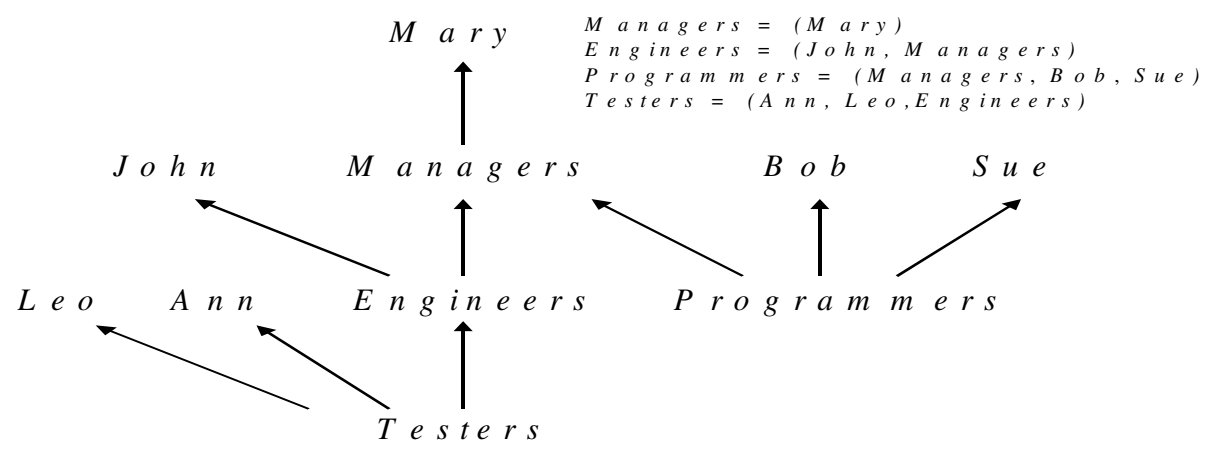

Fig. 2. Set Relations database example

Constraints database (Cdb) contains constraint relations of subject (sets), object (sets), and operation(s). It is used by $R M p$ as a reference to establish a subject from a user's access request. An example entry:

$\left(\mathrm{SS}_{1} ; \mathrm{P}_{1} ; \mathrm{OS}_{1}\right),\left(\mathrm{SS}_{2} ; \mathrm{P}_{2} ; \mathrm{OS}_{2}\right)$ i.e., The combination of subject (sets) $\mathrm{SS}_{1}$, operation(s) $P_{1}$ and object (sets) $O S_{1}$, and the combination of subject (sets) $S S_{2}$, operation(s) $P_{2}$ and object (sets) $O S_{2}$ are mutually excluded.

Privilege database ( $P d b)$ is accessed by SOMp for searching the subject (sets), operation(s) and object (sets) relations. A relation in $P d b$ defines the privilege for the $s u b$ ject (sets). An example of $P d b$ entry is:

Programmers; read, write, execute; file_a i.e., subject set Programmers can read, write, and execute file_a.

History Based Relations database (HBRdb) contains information, which is used in the $A H R D M p$ to perform state transaction of a historical-related policy embedded in the database with the structure:

$s t_{i}$, Event, Actions, $s t_{j}$, where $s t_{i}$ is the current state of the embedded historical access control policies, Event is an authorized access :(subject (sets), operation(s), object (sets)). Actions are sets of actions $\left(a_{l} \ldots \ldots a_{n}\right)$, each $a_{i}=($ dbname, Updates), where the database dbname is the name of one of the $C d b$ or $P d b$ will be updated with the information in Updates. $s t_{j}$ is the next historical state $P M$ will be when the Event occurred.

\subsubsection{Universal Property}

$P M$ can support most major access models, making it a possible to express the corresponding access control policies that these models represent. In order to reduce the support needed to express a multitude of models, we take advantage of previous work which has shown that certain model categories may be simulated or represented by other model categories. For instance, Sandhu has shown in [Sand93] that Latticebased access control models such as the Bell-Lapadula model [BL73] and Biba model [Biba77] may simulate Information Flow polices (including MAC policies) 
and Chinese Wall policies. In $P M$, Lattices are represented by a set implemented by data records in a database and embedded in the PE's $R d b$ as described in section 2.1.1 $R d b$. The relations of the elements of the Lattices are checked in $R M p$ as described in section $2.1 R M p$. This allows us to describe a wide variety of models with a minimum of $P M$ features.

As a second example, $P M$ maintains records for access events and updates databases to reflect changes of the states caused by the events according to the historical policy states that are embedded in $H R B d b$. As indicated in section 2.1.1 $H R B d$, the historical related policy is implemented by a table structure of a database, the process in the AHRDMp tracks the historical state of the policy by referring the information in the table as described in section 2.1 AHRDMp. Therefore, policy models which can be mapped to state transition machine models can also modeled by $P M$; this allows us to support Clark Wilson [CWAC87] and N-person Control policy models, for example.

Gligor, Gavrila and Ferraiolo [GGF98] showed that SOD policies can be enforced either statically or dynamically. We can use either method by employing $P E$ 's $C d b$ as described in section 2.1.1 statically and dynamically. The static $C d b$ constrains relations between subjects, objects and/or operations, and provides the tool for statically separating users of their privileges. The dynamic $C d b$ can be generated by the process of AHRDMp according to the actions stored in the historical policy database and can separate the users' privileges dynamically. Therefore, through the $C d b$ the $P M$ is able to enforce policy models which require either static or dynamic SOD. These policies include Work Flow [AH96] and RBAC.

DAC access control policy can be achieved by appropriately managing the $P d b$ tables associated with each user. The $P d b$ table for each user should be set as a controlled object of the administrator's $P E$. These users will then have sole and total control of their own $P d b$ table, thereby allowing them to delegate the access privilege to other users.

Although a formal proof of correspondence is beyond the scope of this paper, the above paragraphs indicate how the properties and components of $P M$ allow $P M$ to model some major access control models.

\subsection{General Policy Operations (GPO)}

$G P O$ is a set of operations for expressing access control policies. It allows users to specify, together with the authorizations, the policy according to which access control decisions are to be made. GPO has the following components: Basic sets define the basic elements and sets for the GPO. Database query functions represent restrictions or relationships between elements. Administrative operations are used for the administration of the $P M$ databases. Rules are used to keep the transitions in the states of $P M$ during the system operation.

\section{Basic Sets}

A set of basic sets, defines the users, objects, operations and their relations. 
Examples:

$U S=$ the set of user sets, $\left(U S_{1}, \ldots \ldots, U S_{n}\right)$.

$U S_{i}=$ the set of users, $\left(u_{l}, \ldots, u_{n}\right)$, where each user is assigned to user set $U S_{i}$.

\section{Database Query Functions}

There are three types of database query functions:

1. Set query functions, for retrieving relations from $R d b$. Examples: member_of $(s)$ denotes the members of set $s$ ( $s$ is inherited by the members). set_of $(m)$ denotes the sets the member $m$ is assigned to (inherit from). transitive_member_of $(s)$ denotes the transitive members of set $s$. transitive_set_of $(m)$ denotes the transitive sets the member $m$ belongs to.

2. Constraint query functions, for retrieving constrains from $C d b$. Examples: userSet_constrained (s) denotes the constraint relations of the user (set) s. objectSet_constrained (o) denotes the constraint relations of the object (set) o. userSet_permitted ( $s$ ) denotes the permitted relations related to the user (set) s. objectSet_permitted (o) denotes the permitted relations of the object (set) $o$.

3. Process mapping functions, for retrieving information of current process. Examples: operation_request $(u)$ denotes the operation requested by user $u$.

object_request $(u)$ denotes the object requested by user $u$. subject_of $(u)$ denotes the subject associated with $u$ ser $u$. active_userSets ( $(s)$ denotes the set of active user(sets) associated with subject $s$. access $(s, p, o)$ return 1 if subject $s$ is authorized to access object o by operation $p$.

\section{Administrative Operations}

A set of administrative commands. Examples: addMember $(x, y)$, rmMember $(x, y)$, new member $x$ is added/removed to set $y$ in $R d b$.

addConstraints(c), rmConstraintse(c), constraints $c$ is added/removed to $C d b$ database.

addRelations $(c)$, rmRelations $(c)$, relation $c$ is added/removed to $P d b$.

\section{Rules}

The rules represent assumptions about the GPO. Examples:

The member assigned to a given set is exactly the member directly inheriting the given set in Rdb, and the sets assigned to a given member are exactly the sets directly inherited by the member in $R d b$.

Any two user sets assigned to a third user set do not inherit (directly or indirectly) one another in $R d b$.

\subsubsection{PM Composition}

The Administration Operations of GPO allow users of $P M$ to interact with $P M$; they are used to add, remove, and update a $P E$ database entry. Before an Administration 
Operation is executed, GPO will invoke the Database Query Functions built in the $G P O$ to retrieve information required for the operation. $G P O$ will then check the validation of the logics and formats governed by the GPO's Rules. The Rules of GPO guarantee the consistencies of the relations, constraints and privileges of the information stored in the PE databases.

Some of the functions for well-known access control policies can be built in the GPO. Through the Administration Operations, PM users/administrators can implement and manage an access control policy. For example, to add a relation for users in a Separation of Duty (SOD) policy, $P M$ administrators can execute the command:

AddSODusers $(x, y)$, i.e., add separation of duty (mutual exclusive) relation for $u s$ ers $x$ and users $y$.

The Administrative Operations are a key feature of GPO, since they provide the $P M$ administrator a tool for composing new access control policies. Through the GPO's Rule, the information stored in the $P E$ database can be generated and modified without $P M$ administrators having any known access control policy in mind.

The composition capability of $G P O$ supports the important feature of $P M$ that separates the security policies from the security mechanisms.

\subsection{PM Combination}

A key feature of $P M$ is its ability to combine policies. Policies are established in different $P M$ s by implementing their databases and GPOs. When policies are combined, $P M \mathrm{~s}$ are essentially "chained together". In practice, this means that a "User Request" begins at the "first" $P M$, and it is passed along to subsequent $P M$ s for examination. Each $P M$ will in turn examine its own state of completion (i.e., ability to decide if there is enough information to process the request). If it has not reached a state of completion, it will pass the token as an input to the next $P M$ (see Figure 3).

An object can be constrained under more than one policy, for example, a user may get past the MAC policy check, but may still need to be authorized again by other policies say, RBAC for allowing the requested operation to the object. For example, the sequence of checks could be: Can the user read secret information, is the user a doctor, is the patient assigned to the doctor for the operation/procedure. 


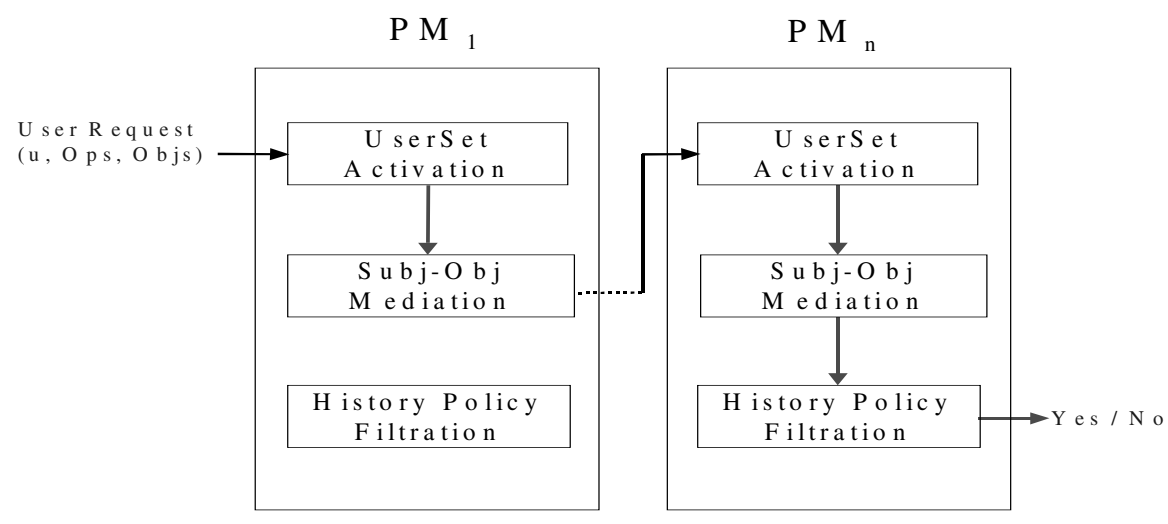

Fig. 3. $P M$ combination

\section{Conclusions}

In this paper, we have demonstrated the concept of constructing universal policy architecture without detailed and formal specification. The issue of formal specification will be the subject of our planned future research. $P M$ functions are executed through sequential phases (as described in Section 2) without recursive operations in the $P M$ functions; therefore it is computable in polynomial time. Since $P M$ 's database is always consistent (no conflict mapping of relations), users' access requests are handled conclusively; that is, they are either authorized or denied.

$P M$ has significant theoretical and practical implications; it is an architecture that is capable of implementing virtually any access control policy. It also helps to promote interoperability and the use of innovative tools for policy generation and visualization that can be built on top of existing access control primitives and scaled to the largest virtual enterprise.

\section{References}

[AH96] Atluri V., Huang W., "An Authorization Model for Workflows". Proceedings of the Fifth European Symposium on Research in Computer Security in Lecture Notes in Computer Science, No 1146, September 1996.

[Ande72] Anderson J. P., "Computer Security Technology Planning Study," ESD_TR_73-51, Vol. 1, Hanscom AFB, Mass., 1972.

[Bark97] Barkley J., "Comparing Simple Role Based Access Control Models and Access Control Lists", Proceedings of the Second ACM Workshop on Role-Based Access Control, November 1997, page 127-132.

[Biba77] Biba K. J., "Integrity Considerations for Secure Computer Systems," ESD-TR-76-372, USAF Electronic Systems Division, Bedford, Mass., April 1977. 
[BL73] Bell D.E., and Lapadula L. J., "Secure Computer Systems: Mathematical Foundations and Model," M74-244, MITRE Corp., Bedford, Mass., 1973.

[BNCW89] Brewer, D., Nash, M. "The Chinese Wall Security Policy." Proc IEEE Symp Security \& Privacy, IEEE Comp Soc Press, 1989, pp 206-214.

[CW87] Clark D. D., and Wilson D. R., "A Comparison of Commercial and Military Security Policies," Proc. of the 1987 IEEE Symposium on Security and Privacy, Oakland, California, 1987, pp.184-194

[CWEO89] Clark D. D., and Wilson D. R., "Evolution of a Model for Computer Integrity", NIST Special Publication 500-168, Appendix A, September 1989

[FCK95] Ferraiolo D. F., Cugini J. A., Kuhn D. R., "Role-Based Access Control (RBAC): Features and Motivations", Proc. of the $11^{\text {th }}$ Annual Conference on Computer Security Applications. IEEE Computer Society Press, Los Alamitos, CA. 1995. [GGF98] Gligor V. D., Gavrila S. I., Ferraiolo D., "On the Formal Definition of Separation-of-Duty Policies and their Composition", In IEEE Symposium on Computer Security and Privacy, April 1998.

[HGPS99] Hale J., Galiasso P., Papa M., Shenoi S., "Security Policy Coordination for Heterogeneous Information Systems", Proc. 15th Annual Computer Security Applications Conference, Applied Computer Security Associates, December 1999.

[ISW97] Irvine C. E., Stemp R., Warren D. F., "Teaching Introductory Computer Security at a Department of Defense University", Naval Postgraduate School Monterey, California, NPSCS-97-002, April 1997

[JSS97] Jajodia S., Samarati P., and Subrahmanian V. S., “A Logical Language for Expressing Authorizations," Proc. IEEE Symp, Oakland, Calif., May 1997.

[JSSB97] Jajodia S., Sammarati P., Subrahmanian V. S., and Bertino E., "A Unified Frame Work for Enforcing Multiple Access Control Policies", Proc. ACM SIGMOD Conf. On Management of Data, Tucson, AZ, May 1997.

[NCSC87] National Computer Security Center (NCSC). "A GUIDE TO UNDERSTANDING DISCRETIONARY ACCESS CONTROL IN TRUSTED SYSTEM", Report NSCD-TG-003 Version1, 30 September 1987.

[NCSC91] National Computer Security Center, "Integrity in Automated information System", $C$ Technical Report 79-91, Library No. S237,254, September 1991.

[NCSC98] National Computer Security Center, "1998 Evaluated Products List", Washington, D.C., U.S. Government Printing Office.

[Sand93] Sandhu R. S., "Lattice-Based Access Control Models", IEEE Computer, Volume 26, Number 11, November 1993, page 9-19.

[SCFY96] Sandhu R. S., Coyne E. J., Feinstein H. L., and Youman C. E., "Role-Based Access Control Models", IEEE Computer, Volume 29, Number 2, February 1996, page 38-47.

[Spencer et a199] Spencer R., Smalley S., Loscocco P., Hibler M., Andersen D., and Lepreau J., "The Flask Security Architecture: System Support for Diverse Security Policies", http://www.cs.utah.edu/fluz/flask, July 1999.

[SZ97] Simon R.T., and Zurko M. E., "Separation of Duty in Role-Based Environments," Proc. of the Computer Security Foundations Workshop X, Rockport, Massachusetts, June 1997. 